\title{
A Further Analysis of the Importance of Systematic Reviews to Computer Science
}

\author{
José Itamar Mendes de Souza Júnior, Valéria Martins Silva, David Nadler Prata, and Patrick Letouze
}

\begin{abstract}
Systematic Review has become an essential scientific method to research in Computer Science, mainly because interdisciplinary studies and scientific research in the Internet are demanding it. This work provides a further analysis on the subject through a systematic review in the domain of Computer Science, which was performed using scientific papers databases relevant to Computer Science, from the years 2006 to 2012 . Our research has shown that $75.5 \%$ of the total number of papers are concentrated in the top 10 journals. Only $54.9 \%$ of the papers declared in the keywords that it was a systematic review, and only $75.7 \%$ declared it in their title. Additionally, our research has verified that $59.8 \%$ of the papers are directly related to Software Engineering.
\end{abstract}

Index Terms-Computer science, technology and education, interdisciplinary research, software engineering, systematic review.

\section{INTRODUCTION}

Previously, we have presented a quantitative analysis of systematic reviews in Computer Science [1] to show its growing importance. In order to do that, a systematic review had to be performed, that is, we have done a systematic review of systematic reviews in Computer Science from 2006 to 2012. Herein, we continue our analysis seeking further insights on the subject.

The importance of systematic reviews in Computer Science as a scientific method had to be assessed, because not only science itself is becoming more interdisciplinary [2], but Computer Science researches are becoming even more interdisciplinary. Especially, when the research is related to the Internet or to human beings.

A systematic review is a method to identify the studies related to a common subject that aims to obtain unbiased knowledge in a comprehensive, systematic and replicable review of the scientific literature. The use of this method in health care studies was already consolidated in the 80's [3] to assess the strength and quality of scientific evidence, especially with regards to clinical recommendations. Its benefits are not only applicable in health researches, but it has also been used in Social Sciences [4], [5].

The interdisciplinary approach in research is also a good

Manuscript received May 2, 2014; revised July 5, 2014.

Patrick Letouze is with the Department of Computer Science, he is the director of the Software Development Nucleus (NDS - Núcleo de Desenvolvimento de Software), the vice-director of the postgraduate program of System's Computational Modeling, and the former director of research of the University. Additionally, he is the elected president of the Committee for Ethics in Research with Human Beings of the University (e-mail: letouze@uft.edu.br,patrick.letouze@gmail.com). enough motive for requiring systematic reviews. It implies in the investigation of two or more fields. That may imply in much more information to investigate than a traditional single field research. This would prevent unwanted bias in the scientific literature review and it could provide, as in the case of Evidence-Based Medicine [3], the assessment of strength and quality of scientific evidence.

Hence, this work is a qualitative analysis of Systematic Reviews in the domain of Computer Science based on a systematic review. For the organization of this paper, we chose IMRAD structure [6]: introduction, methodology, results and discussion. This structure is part of the uniform requirements for manuscripts submitted to biomedicine journals from the International Committee of Medical Journal Editors. The adoption of this framework should facilitate the information storage and retrieval in international databases by search engines for research purposes like systematic reviews and meta-analysis.

\section{Methodology}

A systematic review is a method that gathers a set of similar primary studies that goes through a selection process regarding some specified criteria. This work is based on a systematic review to identify studies which also use the systematic review in the field of Computer Science [1], that is, a quantitative synthesis of other reviews of the literature regarding the Information Technology area.

The Systematic Review performed to support this paper was published in [1], which was based on the study of two other Systematic Reviews in Computer Science, Breivold et al. in [7], and Ampatzoglou and Stamelos in [8], which followed the guidelines presented in [9].

By the way, the steps of a systematic review may be divided in:

- To determine the rules for the review implementation;

- To establish the criteria for inclusion and exclusion;

- To investigate relevant studies;

- To assess the quality, the information extraction, and the synthesis.

The systematic review presented in [1] showed the statistics of publications of systematic reviews in Computer Science between 2006 and 2012. A total of 3,645 articles were examined, out of which 102 were selected, as shown in Table I. Additionally, in Fig. 1, it is shown a direct comparison of the number of selected publications from scientific databases. Both Table I and Fig. 1 presented here are an Errata of our previous work presented in [1].

Therefore, the methodology applied to develop this work was a systematic review with deeper data analysis than 
presented before. Consequently, it may be considered the continuation of our previous work [1].

TABLE I: OUTCOMES FROM RESEARCH GROUNDED ON SCIENTIFIC DATABASES

\begin{tabular}{lcccc}
\hline \hline Database & Total results & $\begin{array}{l}\text { Papers } \\
\text { published in } \\
\text { Journals }\end{array}$ & $\begin{array}{l}\text { Papers in } \\
\text { English }\end{array}$ & $\begin{array}{l}\text { Inclused } \\
\text { papers }\end{array}$ \\
\hline ACM & 536 & 535 & 535 & 9 \\
Compendex & 131 & 41 & 40 & 5 \\
Elsevier & 584 & 584 & 584 & 56 \\
IEEE & 740 & 166 & 166 & 12 \\
ISI Web of & 12 & 4 & 4 & 1 \\
Science & 548 & 514 & 509 & 1 \\
Wiley & 1094 & 333 & 325 & 18 \\
Springer & 3645 & 2178 & 2164 & 102 \\
\hline TOTAL & & & & \\
\hline \hline
\end{tabular}

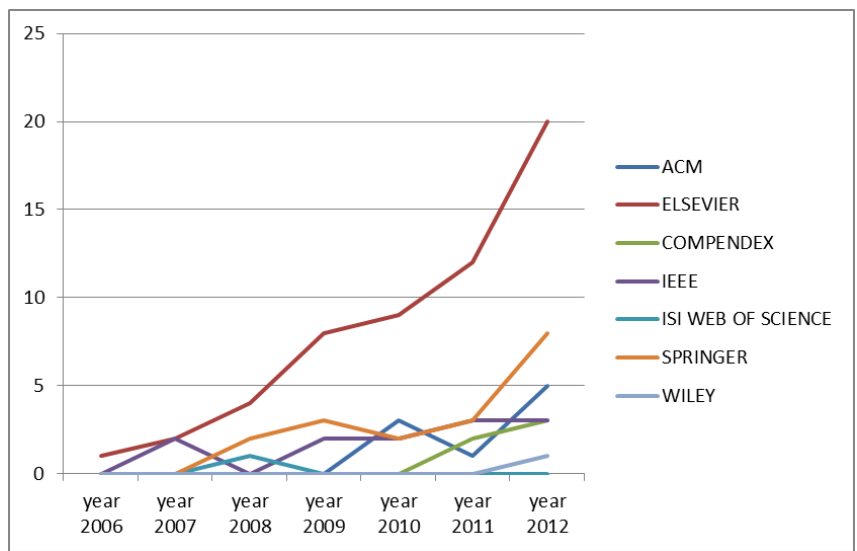

Fig. 1. Comparison among databases.

\section{RESULTS}

The results presented in this section are a deeper analysis of our systematic review presented in [1]. It is based on the data contained in Tables II, III and IV, which comprehend the period between 2006 and 2012.

From Table III - quantity of selected papers published per journal, it is facile to notice that the top 10 journals regarding the quantity of papers represent $75.5 \%$ (77/102). If we consider a quartile analysis, we have that each quartile has 9 journals and they have the following percentage of papers:

- Q1 - $73.5(75 / 102)$;

- $\mathrm{Q} 2-9.8(10 / 102)$;

- $\mathrm{Q} 3-8.8(9 / 102)$;

- Q4-8.8 (9/102).

Additionally, it is quite evident the importance of the journal "Information and Software Technology" of Elsevier, because it responds for $39.2 \%(40 / 102)$ of the selected papers.

From inspection of Table IV, we can observe that the following papers have the words "systematic" and "review" in their titles: [S1], [S2], [S3], [S5], [S6], [S7], [S11], [S12], [S13], [S14], [S15], [S16], [S17], [S18], [S19], [S20], [S21], [S22], [S23], [S24], [S25], [S26], [S27], [S28], [S29], [S30], [S31], [S32], [S33], [S34], [S35], [S36], [S37], [S38], [S39],
[S40], [S41], [S42], [S43], [S44], [S45], [S46], [S47], [S49], [S50], [S51], [S52], [S53], [S55], [S56], [S58], [S63], [S64], [S68], [S70], [S71], [S72], [S73], [S75], [S76], [S77], [S79], [S80], [S82], [S85], [S86], [S87], [S88], [S89], [S91], [S92], [S95], [S97], [S98], [S99], [S100], [S102]. That is, 77 papers out of $102(75.7 \%)$ express in their title that the paper is a systematic review.

From the keyword list - Table V, we observe that the expression "systematic review" appears only 56 times, that is, it appears $54.9 \%(56 / 102)$ of the selected papers.

From Tables III and IV an analysis of terms related to Software Engineering was performed. These lists show that:

- the papers [S3], [S13], [S20], [S30], [S32], [S33], [S34], [S39], [S43], [S44], [S54], [S57], [S58], [S62], [S64], [S71], [S75], [S82], [S90], [S91], [S92], [S96] contain in the keywords "software engineering", that is, 22 of 102 papers $(21.6 \%)$ express in their keywords explicitly that their content is related to Software Engineering;

- and only the papers [S32], [S33], [S34], [S38], [S91], [S96], [S39], [S43], [S44], [S54], [S57], [S58], [S64], [S72], [S82] explicit in their titles that their content is related to Software Engineering, or $14.7 \%$.

However, a thorough examination of the titles and keywords demonstrate that the list of papers related to Software Engineering" is: [S2], [S3], [S6], [S12], [S13], [S14], [S19], [S20], [S22], [S23], [S24], [S28], [S29], [S30], [S32], [S33], [S34], [S36], [S37], [S38], [S39], [S41], [S43], [S44], [S45], [S46], [S47], [S51], [S54], [S56], [S57], [S58], [S59], [S60], [S62], [S64], [S66], [S68], [S71], [S73], [S75], [S77], [S78], [S81], [S82], [S86], [S88], [S90], [S91], [S92], [S96], [S97], [S98], [S99], [S100]. In other words, 55 papers out of $102(53.9 \%)$ are directly related to Software Engineering in their keywords.

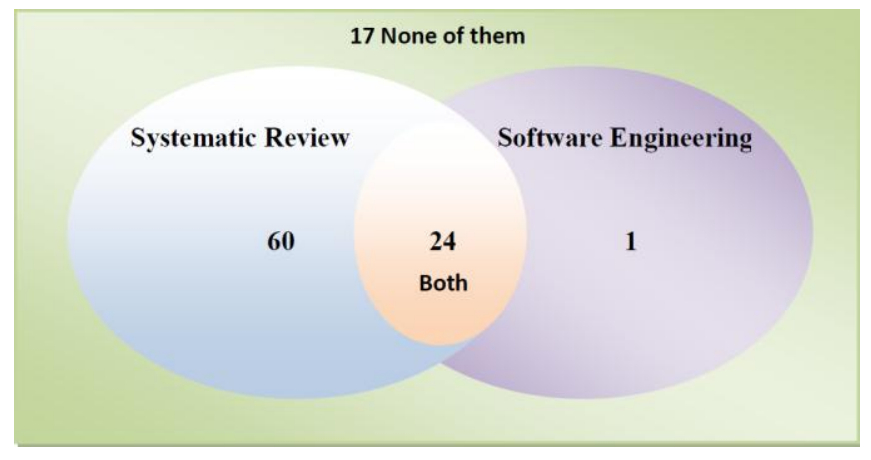

Fig. 2. Relation among papers that declare if they are a systematic review and of software engineering content.

TABLE II: PAPERS RELATED TO SOFTWARE ENGINEERING

\begin{tabular}{llc}
\hline \hline Papers that & $\begin{array}{l}\text { Contain Software } \\
\text { Engineering in the title }\end{array}$ & $\begin{array}{l}\text { Do not contain Software } \\
\text { Engineering in the title }\end{array}$ \\
\hline $\begin{array}{l}\text { Contain Software } \\
\text { Engineering in the key } \\
\text { word }\end{array}$ & 13 & 9 \\
\hline $\begin{array}{l}\text { Do not contain } \\
\text { Software Engineering } \\
\text { in the key word }\end{array}$ & 3 & 36 \\
\hline \hline
\end{tabular}

It is important to notice that it was difficult to determine if the papers [S16], [S21], [S25], [S53], [S70], [S80], [S93], 
[S94], [S101] were related to Software Engineering only through their title and keywords. Then reading their abstract and introduction, we have determined that only the papers [S21], [S25], [S70], [S80], [S93], [S101] were related to Software Engineering. Hence, the total number of papers related to Software Engineering are 61 or $59.8 \%$, which are analyzed in Fig. 2 and Table II.

\section{Discussion}

The number of published papers of systematic review is growing in Computer Science in recent years, Fig. 1 [1]. Our research started with 3,645 papers from which 102 were selected, that is a survival rate of $2.8 \%$. That means that the Systematic Review process is laborious and it requires much attention.

TABLE III: QUANTITY OF SELECTED PAPERS PUBLISHED PER JOURNAL.

\begin{tabular}{|c|c|c|}
\hline Journal & Publisher & qty \\
\hline Information and Software Technology. & Elsevier & 40 \\
\hline Journal of Systems and Software & Elsevier & 9 \\
\hline Empirical Software Engineering & Springer & 6 \\
\hline IEEE Transactions on Software Engineering & IEEE & 6 \\
\hline Software Quality Journal & Springer & 5 \\
\hline Requirements Engineering & Springer & 3 \\
\hline Artificial Intelligence Review & Springer & 2 \\
\hline Computer Standards \& Interfaces & Elsevier & 2 \\
\hline $\begin{array}{c}\text { IEEE Transactions on Engineering } \\
\text { Management }\end{array}$ & IEEE & 2 \\
\hline Journal of Applied Sciences & IGI Global & 2 \\
\hline ACM Computing Surveys & $\mathrm{ACM}$ & 1 \\
\hline ACM Transactions on Computing Education & $\mathrm{ACM}$ & 1 \\
\hline Advanced Engineering Informatics & Elsevier & 1 \\
\hline COMPUTERS INFORMATICS NURSING & Kluwer & 1 \\
\hline Computer Supported Cooperative Work & Springer & 1 \\
\hline IEEE Transactions on Education & IEEE & 1 \\
\hline $\begin{array}{c}\text { Foundations and Trends in Information } \\
\text { Retrieval }\end{array}$ & Elsevier & 1 \\
\hline Future Generation Computer Systems & Elsevier & 1 \\
\hline Health Research Policy and Systems & Springer & 1 \\
\hline Higher-Order and Symbolic Computation & Springer & 1 \\
\hline IEEE Software & IEEE & 1 \\
\hline IEEE Transactions on Software Engineering & IEEE & 1 \\
\hline IET software & IEEE & 1 \\
\hline Intelligent Neuroscience & Hindawi. & 1 \\
\hline $\begin{array}{l}\text { International Journal of Enterprise Information } \\
\text { Systems }\end{array}$ & IGI Global & 1 \\
\hline $\begin{array}{l}\text { International Journal of Information and } \\
\text { Computer Security } \\
\end{array}$ & Inderscience & 1 \\
\hline $\begin{array}{c}\text { International Journal of Metadata, Semantics } \\
\text { and Ontologies }\end{array}$ & Inderscience & 1 \\
\hline Journal of Database Management & IGI Global & 1 \\
\hline Journal of Network and Computer Applications & Elsevier & 1 \\
\hline Journal of Network and Computer Applications & Elsevier & 1 \\
\hline $\begin{array}{l}\text { Journal of the American Society for Information } \\
\text { Science and Technology }\end{array}$ & Wiley & 1 \\
\hline Multimedia Systems & Springer & 1 \\
\hline Procedia Technology & Elsevier & 1 \\
\hline Journal of Systems and Control Engineering & Domain analysis & 1 \\
\hline Service Oriented Computing and Applications & Springer & 1 \\
\hline
\end{tabular}

Our research has shown that the top 10 journals that had published more systematic reviews papers in Computer Science represents $75.5 \%$ of the total number of papers. The number one journal regarding that - "Information and Software Technology", is responsible for $39.2 \%$ of the total number of papers. Only $54.9 \%$ of the papers declared in the keywords that it was a systematic review, and only $75.7 \%$ declared it in their title. Additionally, our research has verified that $59.8 \%$ are directly related to Software Engineering.

Consequently, as in [6], we recommend that every "Systematic Review" paper in Computer Science should declare it in their title and keywords. Another recommendation is to declare that the paper is related to "Software Engineering" should be explicit in the keywords. Both statements would facilitate and improve the results of systematic reviews in software engineering.

Finally, it is important to report that in the Department of Computer Science of the Federal University of Tocantins (UFT - Universidade Federal do Tocantins), Systematic Review has been taught in the discipline of Scientific Methodology for the last two years resulting in the publication of papers such as in [1], [10] and this paper itself, i.e., we have been teaching systematic review for both graduate and undergraduate students in an effective way, which suggests further studies of efficiency in the learning process.

TABLE IV: LIST OF SELECTED PAPERS.

\begin{tabular}{|c|c|}
\hline [S1] & $\begin{array}{l}\text { Philip Woodall and Pearl Brereton. 2010. A systematic literature } \\
\text { review of inference strategies.Int. J. Inf. Comput. Secur. 4, } 2 \\
\text { (August 2010), 99-117. DOI=10.1504/IJICS.2010.034813 } \\
\text { http://dx.doi.org/10.1504/IJICS.2010.034813 }\end{array}$ \\
\hline [S2] & $\begin{array}{l}\text { Sonia Montagud, Silvia Abrahão, and Emilio Insfran. 2012. A } \\
\text { systematic review of quality attributes and measures for software } \\
\text { product lines. Software Quality Control 20, 3-4 (September 2012), } \\
\text { 425-486. DOI=10.1007/s11219-011-9146-7 } \\
\text { http://dx.doi.org/10.1007/s11219-011-9146-7 }\end{array}$ \\
\hline [S3] & $\begin{array}{l}\text { Marial\&\\
#45;Cruz Valiente. 2010. A systematic review of research } \\
\text { on integration of ontologies with the model\\
&\\
#45; driven } \\
\text { approach. Int. J. Metadata Semant. Ontologies 5, } 2 \text { (May 2010), } \\
\text { 134-150. DOI=10.1504/IJMSO.2010.033283 } \\
\text { http://dx.doi.org/10.1504/IJMSO.2010.033283 }\end{array}$ \\
\hline [S4] & $\begin{array}{l}\text { E. W. Lang, A. M. Tomé, I. R. Keck, J. M. Górriz-Sáez, and C. G. } \\
\text { Puntonet. 2012. Brain connectivity analysis: a short survey. Intell. } \\
\text { Neuroscience 2012, Article } 8 \text { (January 2012), } 1 \text { pages. } \\
\text { DOI=10.1155/2012/412512 } \\
\text { http://dx.doi.org/10.1155/2012/412512 }\end{array}$ \\
\hline [S5] & $\begin{array}{l}\text { Barbara A. Kitchenham, Pearl Brereton, Mark Turner, Mahmood } \\
\text { K. Niazi, Stephen Linkman, Rialette Pretorius, and David Budgen. } \\
\text { 2010. Refining the systematic literature review process--two } \\
\text { participant-observer case studies. Empirical Softw. Engg. 15, } 6 \\
\text { (December 2010), 618-653. DOI=10.1007/s10664-010-9134-8 } \\
\text { http://dx.doi.org/10.1007/s10664-010-9134-8 }\end{array}$ \\
\hline [S6] & $\begin{array}{l}\text { Mario Piattini, Geert Poels, Marcela Genero, Ana M. } \\
\text { Fernández-Saez, and H. James Nelson. 2011. Research Review: A } \\
\text { Systematic Literature Review on the Quality of UML Models. J. } \\
\text { Database Manage. 22, } 3 \text { (July 2011), 46-70. } \\
\text { DOI=10.4018/jdm.2011070103 } \\
\text { http://dx.doi.org/10.4018/jdm.2011070103 }\end{array}$ \\
\hline [S7] & $\begin{array}{l}\text { Sudhanshu Tyagi and Neeraj Kumar. 2012. Review: A systematic } \\
\text { review on clustering and routing techniques based upon LEACH } \\
\text { protocol for wireless sensor networks. J. Netw. Comput. Appl. 36, } \\
2 \text { (March 2012), 623-645. DOI=10.1016/j.jnca.2012.12.001 } \\
\text { http://dx.doi.org/10.1016/j.jnca.2012.12.001 }\end{array}$ \\
\hline [S8] & $\begin{array}{l}\text { Boris Danev, Davide Zanetti, and Srdjan Capkun. 2012. On } \\
\text { physical-layer identification of wireless devices. ACM Comput. } \\
\text { Surv. 45, 1, Article } 6 \text { (December 2012), } 29 \text { pages. } \\
\text { DOI=10.1145/2379776.2379782 } \\
\text { http://doi.acm.org/10.1145/2379776.2379782 }\end{array}$ \\
\hline [S9] & $\begin{array}{l}\text { Michael J. O'Grady. 2012. Practical Problem-Based Learning in } \\
\text { Computing Education. Trans. Comput. Educ. 12, 3, Article } 10 \\
\text { (July 2012), } 16 \text { pages. DOI=10.1145/2275597.2275599 }\end{array}$ \\
\hline
\end{tabular}




\begin{tabular}{|c|c|}
\hline & http://doi.acm.org/10.1145/2 \\
\hline [S10] & $\begin{array}{l}\text { Lerina Aversano, Carmine Grasso, Maria Tortorella, A Literature } \\
\text { Review of Business/IT Alignment Strategies, Procedia } \\
\text { Technology, Volume 5, 2012, Pages 462-474, ISSN 2212-0173, } \\
\text { http://dx.doi.org/10.1016/j.protcy.2012.09.051. }\end{array}$ \\
\hline [S11] & $\begin{array}{l}\text { Sarah Heckman and Laurie Williams. 2011. A systematic literature } \\
\text { review of actionable alert identification techniques for automated } \\
\text { static code analysis. Inf. Softw. Technol. 53, } 4 \text { (April 2011), } \\
\text { 363-387. DOI=10.1016/j.infsof.2010.12.007 } \\
\text { http://dx.doi.org/10.1016/j.infsof.2010.12.007 }\end{array}$ \\
\hline [S12] & $\begin{array}{l}\text { Lars M. Karg, Michael Grottke, and Arne Beckhaus. 2011. A } \\
\text { systematic literature review of software quality cost research. J. } \\
\text { Syst. Softw. } 84,3 \text { (March 2011), 415-427. } \\
\text { DOI=10.1016/j.jss.2010.11.904 } \\
\text { http://dx.doi.org/10.1016/j.jss.2010.11.904 }\end{array}$ \\
\hline [S13] & $\begin{array}{l}\text { Carla Pacheco and Ivan Garcia. 2012. A systematic literature } \\
\text { review of stakeholder identification methods in requirements } \\
\text { elicitation. J. Syst. Softw. 85, } 9 \text { (September 2012), 2171-2181. } \\
\text { DOI=10.1016/j.jss.2012.04.075 } \\
\text { http://dx.doi.org/10.1016/j.jss.2012.04.075 }\end{array}$ \\
\hline [S14] & $\begin{array}{l}\text { Gursimran Singh Walia and Jeffrey C. Carver. 2009. A systematic } \\
\text { literature review to identify and classify software requirement } \\
\text { errors. Inf. Softw. Technol. 51, } 7 \text { (July 2009), 1087-1109. } \\
\text { DOI=10.1016/j.infsof.2009.01.004 } \\
\text { http://dx.doi.org/10.1016/j.infsof.2009.01.004 }\end{array}$ \\
\hline [S15] & $\begin{array}{l}\text { Gerald Holl, Paul Grünbacher, and Rick Rabiser. 2012. A } \\
\text { systematic review and an expert survey on capabilities supporting } \\
\text { multi product lines. Inf. Softw. Technol. 54, 8 (August 2012), } \\
\text { 828-852. DOI=10.1016/j.infsof.2012.02.002 } \\
\text { http://dx.doi.org/10.1016/j.infsof.2012.02.002 }\end{array}$ \\
\hline [S16] & $\begin{array}{l}\text { Eladio DomiNguez, Beatriz PéRez, ÁNgel L. Rubio, and MarıA A. } \\
\text { Zapata. 2012. A systematic review of code generation proposals } \\
\text { from state machine specifications. Inf. Softw. Technol. 54, } 10 \\
\text { (October 2012), 1045-1066. DOI=10.1016/j.infsof.2012.04.008 } \\
\text { http://dx.doi.org/10.1016/j.infsof.2012.04.008 }\end{array}$ \\
\hline [S17] & $\begin{array}{l}\text { Martin Ivarsson and Tony Gorschek. 2011. A method for } \\
\text { evaluating rigor and industrial relevance of technology } \\
\text { evaluations. Empirical Softw. Engg. 16, } 3 \text { (June 2011), 365-395. } \\
\text { DOI=10.1007/s10664-010-9146-4 } \\
\text { http://dx.doi.org/10.1007/s10664-010-9146-4 }\end{array}$ \\
\hline [S18] & $\begin{array}{l}\text { Liana Barachisio Lisboa, Vinicius Cardoso Garcia, Daniel } \\
\text { Lucrédio, Eduardo Santana de Almeida, Silvio Romero de Lemos } \\
\text { Meira, and Renata Pontin de Mattos Fortes. 2010. A systematic } \\
\text { review of domain analysis tools. Inf. Softw. Technol. 52, } 1 \\
\text { (January 2010), 1-13. DOI=10.1016/j.infsof.2009.05.001 } \\
\text { http://dx.doi.org/10.1016/j.infsof.2009.05.001 }\end{array}$ \\
\hline [S19] & $\begin{array}{l}\text { Lianping Chen, Muhammad Ali Babar, and Nour Ali. } 2009 . \\
\text { Variability management in software product lines: a systematic } \\
\text { review. In Proceedings of the 13th International Software Product } \\
\text { Line Conference (SPLC '09). Carnegie Mellon University, } \\
\text { Pittsburgh, PA, USA, 81-90. }\end{array}$ \\
\hline [S20] & $\begin{array}{l}\text { HÖST, Martin; ORUČEVIĆ-ALAGIĆ, Alma. A systematic review } \\
\text { of research on open source software in commercial software } \\
\text { product development.Information and Software Technology, v. 53, } \\
\text { n. } 6, \text { p. } 616-624,2011 .\end{array}$ \\
\hline [S21] & $\begin{array}{l}\text { Wasif Afzal, Richard Torkar, and Robert Feldt. 2009. A systematic } \\
\text { review of search-based testing for non-functional system } \\
\text { properties. Inf. Softw. Technol. 51, } 6 \text { (June 2009), 957-976. } \\
\text { DOI=10.1016/j.infsof.2008.12.005 } \\
\text { http://dx.doi.org/10.1016/j.infsof.2008.12.005 }\end{array}$ \\
\hline$[\mathrm{S} 22]$ & $\begin{array}{l}\text { Daniel Mellado, Carlos Blanco, Luis E. Sánchez, and Eduardo } \\
\text { Fernández-Medina. 2010. A systematic review of security } \\
\text { requirements engineering. Comput. Stand. Interfaces 32, } 4 \text { (June } \\
\text { 2010), 153-165. DOI=10.1016/j.csi.2010.01.006 } \\
\text { http://dx.doi.org/10.1016/j.csi.2010.01.006 }\end{array}$ \\
\hline$[\mathrm{S} 23]$ & $\begin{array}{l}\text { Hongyu Pei Breivold, Ivica Crnkovic, and Magnus Larsson. } 2012 . \\
\text { A systematic review of software architecture evolution } \\
\text { research. Inf. Softw. Technol. 54, } 1 \text { (January 2012), 16-40. } \\
\text { DOI=10.1016/j.infsof.2011.06.002 } \\
\text { http://dx.doi.org/10.1016/j.infsof.2011.06.002 }\end{array}$ \\
\hline
\end{tabular}

Ali Shahrokni, Robert Feldt, A systematic review of software robustness, Information and Software Technology, Volume 55, Issue 1, January 2012, Pages 1-17, ISSN 0950-5849,

[S24] http://dx.doi.org/10.1016/j.infsof.2012.06.002

(http://www.sciencedirect.com/science/article/pii/S095058491200 1048)

Emelie Engström, Per Runeson, and Mats Skoglund. 2010. A systematic review on regression test selection techniques. Inf.

[S25] Softw. Technol. 52, 1 (January 2010), 14-30.

DOI=10.1016/j.infsof.2009.07.001

http://dx.doi.org/10.1016/j.infsof.2009.07.001

Amir Hossein Ghapanchi and Aybuke Aurum. 2011. Antecedents

to IT personnel's intentions to leave: A systematic literature

[S26] review. J. Syst. Softw. 84, 2 (February 2011), 238-249.

DOI $=10.1016 /$ j.jss.2010.09.022

http://dx.doi.org/10.1016/j.jss.2010.09.022

Carlos Blanco, Joaqu\\&\\#237;n Lasheras, Eduardo

Fern \\&\\#225;ndez-Medina, Rafael Valencia-Garcl\&\\#237;a, and

Ambrosio Toval. 2011. Basis for an integrated security ontology

[S27] according to a systematic review of existing proposals. Comput.

Stand. Interfaces 33, 4 (June 2011), 372-388.

DOI $=10.1016 /$ j.csi.2010.12.002

http://dx.doi.org/10.1016/j.csi.2010.12.002

Byron J. Williams and Jeffrey C. Carver. 2010. Characterizing

software architecture changes: A systematic review. Inf. Softw.

[S28] Technol. 52, 1 (January 2010), 31-51.

DOI=10.1016/j.infsof.2009.07.002

http://dx.doi.org/10.1016/j.infsof.2009.07.002

Parastoo Mohagheghi, Vegard Dehlen, and Tor Neple. 2009

Definitions and approaches to model quality in model-based

software development - A review of literature. Inf. Softw.

[S29] Technol. 51, 12 (December 2009), 1646-1669.

DOI=10.1016/j.infsof.2009.04.004

http://dx.doi.org/10.1016/j.infsof.2009.04.004

Tore Dybå and Torgeir Dingsøyr. 2008. Empirical studies of agile

software development: A systematic review. Inf. Softw.

[S30] Technol. 50, 9-10 (August 2008), 833-859.

DOI $=10.1016 /$ j.infsof .2008 .01 .006

http://dx.doi.org/10.1016/j.infsof.2008.01.006

Antoni Lluís Mesquida, Antonia Mas, Esperança Amengual, and Jose A. Calvo-Manzano. 2012. IT Service Management Process

[S31] Improvement based on ISO/IEC 15504: A systematic review. Inf. Softw. Technol. 54, 3 (March 2012), 239-247.

DOI $=10.1016 /$ j.infsof .2011 .11 .002

http://dx.doi.org/10.1016/j.infsof.2011.11.002

Finn Olav Bj\\&\\#248;rnson and Torgeir Dings\\&\\#248;yr. 2008

Knowledge management in software engineering: A systematic

[S32] review of studied concepts, findings and research methods

used. Inf. Softw. Technol. 50, 11 (October 2008), 1055-1068.

DOI $=10.1016 /$ j.infsof.2008.03.006

http://dx.doi.org/10.1016/j.infsof.2008.03.006

Pearl Brereton, Barbara A. Kitchenham, David Budgen, Mark Turner, and Mohamed Khalil. 2007. Lessons from applying the systematic literature review process within the software

[S33] systering domain. J. Syst. Softw. 80, 4 (April 2007), 571-583. DOI $=10.1016 /$ j.jss.2006.07.009

http://dx.doi.org/10.1016/j.jss.2006.07.009

Sarah Beecham, Nathan Baddoo, Tracy Hall, Hugh Robinson, and

Helen Sharp. 2008. Motivation in Software Engineering: A

[S34] systematic literature review. Inf. Softw. Technol. 50, 9-10 (August 2008), 860-878. DOI=10.1016/j.infsof.2007.09.004

http://dx.doi.org/10.1016/j.infsof.2007.09.004

Rafael Prikladnicki and Jorge Luis Nicolas Audy. 2010. Process models in the practice of distributed software development: A

[S35] systematic review of the literature. Inf. Softw. Technol.52, 8 (August 2010), 779-791. DOI=10.1016/j.infsof.2010.03.009 http://dx.doi.org/10.1016/j.infsof.2010.03.009

Andréa Magalhães Magdaleno, Cláudia Maria Lima Werner, and

Renata Mendes de Araujo. 2012. Reconciling software

development models: A quasi-systematic review. J. Syst

[S36] Softw. 85, 2 (February 2012), 351-369.

DOI $=10.1016 /$ j.jss. 2011.08 .028

http://dx.doi.org/10.1016/j.jss.2011.08.028 


\begin{tabular}{|c|c|}
\hline [S37] & $\begin{array}{l}\text { Vander Alves, Nan Niu, Carina Alves, and George } \\
\text { Valenl\&l\#231;a. 2010. Requirements engineering for software } \\
\text { product lines: A systematic literature review. Inf. Softw. } \\
\text { Technol. 52, 8 (August 2010), 806-820. } \\
\text { DOI=10.1016/j.infsof.2010.03.014 } \\
\text { http://dx.doi.org/10.1016/j.infsof.2010.03.014 }\end{array}$ \\
\hline [S38] & $\begin{array}{l}\text { Wasif Afzal and Richard Torkar. 2011. Review: On the application } \\
\text { of genetic programming for software engineering predictive } \\
\text { modeling: A systematic review. Expert Syst. Appl. 38, } 9 \\
\text { (September 2011), 11984-11997. } \\
\text { DOI=10.1016/j.eswa.2011.03.041 } \\
\text { http://dx.doi.org/10.1016/j.eswa.2011.03.041 }\end{array}$ \\
\hline [S39] & $\begin{array}{l}\text { Fabio Q. B. da Silva, André L. M. Santos, Sérgio Soares, A. César } \\
\text { C. França, Cleviton V. F. Monteiro, and Felipe Farias Maciel. } \\
\text { 2011. Six years of systematic literature reviews in software } \\
\text { engineering: An updated tertiary study. Inf. Softw. Technol. 53, } 9 \\
\text { (September 2011), 899-913. DOI=10.1016/j.infsof.2011.04.004 } \\
\text { http://dx.doi.org/10.1016/j.infsof.2011.04.004 }\end{array}$ \\
\hline [S40] & $\begin{array}{l}\text { Dhavleesh Rattan, Rajesh Kumar Bhatia, Maninder } \\
\text { Singh: Software clone detection: A systematic review. Information } \\
\text { \& Software Technology 55(7): 1165-1199 (2012) }\end{array}$ \\
\hline [S41] & $\begin{array}{l}\text { Danijel Radjenović, Marjan Heričko, Richard Torkar, Aleš } \\
\text { Živkovič, Software fault prediction metrics: A systematic literature } \\
\text { review, Information and Software Technology, Volume 55, Issue } \\
\text { 8, August 2012, Pages 1397-1418, ISSN 0950-5849, } \\
\text { http://dx.doi.org/10.1016/j.infsof.2012.02.009.(http://www.scienc } \\
\text { edirect.com/science/article/pii/S0950584913000426) }\end{array}$ \\
\hline [S42] & $\begin{array}{l}\text { Jianfeng Wen, Shixian Li, Zhiyong Lin, Yong Hu, and Changqin } \\
\text { Huang. 2012. Systematic literature review of machine learning } \\
\text { based software development effort estimation models. Inf. Softw. } \\
\text { Technol. 54, 1 (January 2012), 41-59. } \\
\text { DOI=10.1016/j.infsof.2011.09.002 } \\
\text { http://dx.doi.org/10.1016/j.infsof.2011.09.002 }\end{array}$ \\
\hline [S43] & $\begin{array}{l}\text { Barbara Kitchenham, O. Pearl Brereton, David Budgen, Mark } \\
\text { Turner, John Bailey, and Stephen Linkman. 2009. Systematic } \\
\text { literature reviews in software engineering - A systematic literature } \\
\text { review. Inf. Softw. Technol. 51, 1 (January 2009), 7-15. } \\
\text { DOI=10.1016/j.infsof.2008.09.009 } \\
\text { http://dx.doi.org/10.1016/j.infsof.2008.09.009 }\end{array}$ \\
\hline [S44] & $\begin{array}{l}\text { Barbara Kitchenham, Rialette Pretorius, David Budgen, O. Pearl } \\
\text { Brereton, Mark Turner, Mahmood Niazi, and Stephen Linkman. } \\
\text { 2010. Systematic literature reviews in software engineering - A } \\
\text { tertiary study. Inf. Softw. Technol. 52, } 8 \text { (August 2010), 792-805. } \\
\text { DOI=10.1016/j.infsof.2010.03.006 } \\
\text { http://dx.doi.org/10.1016/j.infsof.2010.03.006 }\end{array}$ \\
\hline [S45] & $\begin{array}{l}\text { Mark Staples and Mahmood Niazi. 2008. Systematic review: } \\
\text { Systematic review of organizational motivations for adopting } \\
\text { CMM-based SPI. Inf. Softw. Technol. 50, 7-8 (June 2008), } \\
\text { 605-620. DOI=10.1016/j.infsof.2007.07.003 } \\
\text { http://dx.doi.org/10.1016/j.infsof.2007.07.003 }\end{array}$ \\
\hline [S46] & $\begin{array}{l}\text { Roberto dos Santos Rocha, Marcelo Fantinato, The use of software } \\
\text { product lines for business process management: A systematic } \\
\text { literature review, Information and Software Technology, Volume } \\
\text { 55, Issue 8, August 2012, Pages 1355-1373, ISSN 0950-5849, } \\
\text { http://dx.doi.org/10.1016/j.infsof.2012.02.007. } \\
\text { (http://www.sciencedirect.com/science/article/pii/S095058491300 } \\
\text { 0402) }\end{array}$ \\
\hline [S47] & $\begin{array}{l}\text { Sara Mahdavi-Hezavehi, Matthias Galster, and Paris Avgeriou. } \\
\text { 2012. Variability in quality attributes of service-based software } \\
\text { systems: A systematic literature review. Inf. Softw. Technol. 55, } 2 \\
\text { (February 2012), 320-343. DOI=10.1016/j.infsof.2012.08.010 } \\
\text { http://dx.doi.org/10.1016/j.infsof.2012.08.010 }\end{array}$ \\
\hline [S48] & $\begin{array}{l}\text { Jacques Wainer, Claudia G. Novoa Barsottini, Danilo Lacerda, and } \\
\text { Leandro Rodrigues Magalhl\&l\#227; es de Marco. 2009. Empirical } \\
\text { evaluation in Computer Science research published by ACM. Inf. } \\
\text { Softw. Technol. 51, } 6 \text { (June 2009), 1081-1085. } \\
\text { DOI=10.1016/j.infsof.2009.01.002 } \\
\text { http://dx.doi.org/10.1016/j.infsof.2009.01.002 }\end{array}$ \\
\hline [S49 & $\begin{array}{l}\text { Gordon V. Cormack. 2008. Email Spam Filtering: A Systemat } \\
\text { Review. Found. Trends Inf. Retr.1, } 4 \text { (April 2008), 335-455. }\end{array}$ \\
\hline
\end{tabular}

\begin{tabular}{|l|l|}
\hline & DOI=10.1561/1500000006 http://dx.doi.org/10.1561/1500000006 \\
\hline \multirow{5}{*}{ [S50] } & $\begin{array}{l}\text { Ahmed Patel, Mona Taghavi, Kaveh Bakhtiyari, and Joaquim } \\
\text { Celestino JúNior. 2012. Review: An intrusion detection and } \\
\text { prevention system in cloud computing: A systematic review. J. } \\
\text { Netw. Comput. Appl. 36, 1 (January 2012), 25-41. } \\
\text { DOI=10.1016/j.jnca.2012.08.007 } \\
\text { http://dx.doi.org/10.1016/j.jnca.2012.08.007 }\end{array}$ \\
\hline
\end{tabular}

Francisco J. Lucas, Fernando Molina, and Ambrosio Toval. 2009. A systematic review of UML model consistency management. Inf. [S51] Softw. Technol. 51, 12 (December 2009), 1631-1645.

DOI=10.1016/j.infsof.2009.04.009

http://dx.doi.org/10.1016/j.infsof.2009.04.009

Mikael Svahnberg, Tony Gorschek, Robert Feldt, Richard Torkar,

Saad Bin Saleem, and Muhammad Usman Shafique. 2010. A

[S52] systematic review on strategic release planning models.Inf. Softw. Technol. 52, 3 (March 2010), 237-248.

DOI $=10.1016 / \mathrm{j}$.infsof.2009.11.006

http://dx.doi.org/10.1016/j.infsof.2009.11.006

Øyvind Hauge, Claudia Ayala, and Reidar Conradi. 2010.

Adoption of open source software in software-intensive

[S53] organizations - A systematic literature review. Inf. Softw.

Technol. 52, 11 (November 2010), 1133-1154.

DOI $=10.1016 /$ j.infsof 2010.05 .008

http://dx.doi.org/10.1016/j.infsof.2010.05.008

He Zhang, Muhammad Ali Babar, and Paolo Tell. 2011.

Identifying relevant studies in software engineering. Inf. Softw.

[S54] Technol. 53, 6 (June 2011), 625-637.

DOI $=10.1016 /$ j.infsof 2010.12 .010

http://dx.doi.org/10.1016/j.infsof.2010.12.010

IváN Santiago, ÁLvaro JiméNez, Juan Manuel Vara, Valeria De

Castro, VeróNica A. Bollati, and Esperanza Marcos. 2012.

Model-Driven Engineering as a new landscape for traceability

[S55] management: A systematic literature review. Inf. Softw.

Technol. 54, 12 (December 2012), 1340-1356.

DOI=10.1016/j.infsof.2012.07.008

http://dx.doi.org/10.1016/j.infsof.2012.07.008

Stephen Lane and Ita Richardson. 2011. Process models for service-based applications: A systematic literature review. Inf.

[S56] Softw. Technol. 53, 5 (May 2011), 424-439.

DOI $=10.1016 /$ j.infsof. 2010.12 .005

http://dx.doi.org/10.1016/j.infsof.2010.12.005

Daniela S. Cruzes and Tore Dybå. 2011. Research synthesis in

[S57] software engineering: A tertiary study. Inf. Softw. Technol. 53, 5 (May 2011), 440-455. DOI=10.1016/j.infsof.2011.01.004 http://dx.doi.org/10.1016/j.infsof.2011.01.004

Apostolos Ampatzoglou and Ioannis Stamelos. 2010. Software engineering research for computer games: A systematic

[S58] review. Inf. Softw. Technol. 52, 9 (September 2010), 888-901. DOI=10.1016/j.infsof.2010.05.004

http://dx.doi.org/10.1016/j.infsof.2010.05.004

Barney, S., Petersen, K., Svahnberg, M., Aurum, A., \& Barney, H.

[S59] (2012). Software quality trade-offs: A systematic map. Information and Software Technology, 54(7), 651-662.

Marcos Palacios, Jos\\&\\#233; Garcl\&\\#237;a-Fanjul, and Javier Tuya. 2011. Testing in Service Oriented Architectures with

[S60] dynamic binding: A mapping study. Inf. Softw. Technol. 53, 3

(March 2011), 171-189. DOI=10.1016/j.infsof.2010.11.014 http://dx.doi.org/10.1016/j.infsof.2010.11.014

Henry Edison, Nauman Bin Ali, and Richard Torkar. 2012

Towards innovation measurement in the software industry. J. Syst.

[S61] Softw. 86, 5 (May 2012), 1390-1407.

DOI $=10.1016 / j$ j.jss.2012.01.013

http://dx.doi.org/10.1016/j.jss.2012.01.013

Fabio Q. B. da Silva and A. César C. França. 2012. Towards understanding the underlying structure of motivational factors for

[S62]

software engineers to guide the definition of motivational

programs. J. Syst. Softw. 85, 2 (February 2012), 216-226.

DOI $=10.1016 /$ j.jss.2010.12.017

http://dx.doi.org/10.1016/j.jss.2010.12.017 
Siffat Ullah Khan, Mahmood Niazi, and Rashid Ahmad. 2011. [S63] Factors influencing clients in the selection of offshore software outsourcing vendors: An exploratory study using a systematic literature review. J. Syst. Softw. 84, 4 (April 2011), 686-699. $\mathrm{DOI}=10.1016 / \mathrm{j}$.jss.2010.12.010

http://dx.doi.org/10.1016/j.jss.2010.12.010

Jorge Calmon de Almeida Biolchini, Paula Gomes Mian, Ana Candida Cruz Natali, Tayana Uch $\ \ \$ 244 ;$; Conte, and Guilherme

[S64] Horta Travassos. 2007. Scientific research ontology to support systematic review in software engineering. Adv. Eng. Inform. 21, 2 (April 2007), 133-151. DOI=10.1016/j.aei.2006.11.006 http://dx.doi.org/10.1016/j.aei.2006.11.006

Jingcheng Gao, Yang Xiao, Jing Liu, Wei Liang, and C.L. Philip Chen. 2012. A survey of communication/networking in Smart

[S65] Grids. Future Gener. Comput. Syst. 28, 2 (February 2012), 391-404. DOI=10.1016/j.future.2011.04.014 http://dx.doi.org/10.1016/j.future.2011.04.014

MAGABLEH, Aws A.; SHUKUR, Zarina; ALI, Noorazean Mohd.

[S66] An Aspectual UML Modelling T001. Journal of Applied Sciences, v. 12, n. 21, p. 2182-2194, 2012.

Veres, S. M., Molnar, L., Lincoln, N. K., \& Morice, C. P. (2011). Autonomous vehicle control systems - a review of decision

[S67] making. Proceedings of the Institution of Mechanical Engineers, Part I: Journal of Systems and Control Engineering, 225(2), 155-195.

\section{El-Attar, M., \& Miller, J. (2012). Constructing high quality use}

[S68] case models: a systematic review of current practices. Requirements Engineering, 17(3), 187-201.

Ahmad, M. N., Zakaria, N. H., \& Sedera, D. (2011).

[S69] Ontology-based knowledge management for enterprise systems. International Journal of Enterprise Information Systems (IJEIS), 7(4), 64-90.

Systematic review on aspect-oriented UML modeling: A complete aspectual UML modeling framework Magableh, Aws (Department of Software Engineering, Faculty of Computer Science and [S70] Information Technology, Universiti Kebangsaan Malaysia (UKM), 34600, Kajang, Malaysia); Shukur, Zarina; Ali, Noorazean Mohd Source: Journal of Applied Sciences, v 13, n 1, p 1-13, 2012

Brereton, P. (2011). A study of computing undergraduates

[S71] undertaking a systematic literature review. Education, IEEE Transactions on, 54(4), 558-563.

Hall, T., Beecham, S., Bowes, D., Gray, D., \& Counsell, S. (2012). A systematic literature review on fault prediction performance in

[S72] Aftware engineering. Software Engineering, IEEE Transactions on, 38(6), 1276-1304.

Jorgensen, M., \& Shepperd, M. (2007). A systematic review of

[S73] software development cost estimation studies. Software Engineering, IEEE Transactions on, 33(1), 33-53.

Cornelissen, B., Zaidman, A., Van Deursen, A., Moonen, L., \&

Koschke, R. (2009). A systematic survey of program

[S74] Koschehension through dynamic analysis. Software Engineering, IEEE Transactions on, 35(5), 684-702.

Kitchenham, B. A., Mendes, E., \& Travassos, G. H. (2007). Cross

[S75] versus within-company cost estimation studies: A systematic review. Software Engineering, IEEE Transactions on, 33(5), 316-329.

Salleh, N., Mendes, E., \& Grundy, J. (2011). Empirical studies of

[S76] pair programming for CS/SE teaching in higher education: A systematic literature review. Software Engineering, IEEE Transactions on, 37(4), 509-525.

Unterkalmsteiner, M., Gorschek, T., Islam, A. M., Cheng, C. K., Permadi, R. B., \& Feldt, R. (2012). Evaluation and measurement of

[S77] software process improvement-A systematic literature review. Software Engineering, IEEE Transactions on, 38(2), 398-424.

Persson, J. S., Mathiassen, L., Boeg, J., Madsen, T. S., \& Steinson,

[S78] F. (2009). Managing risks in distributed software projects: an integrative framework. Engineering Management, IEEE Transactions on, 56(3), 508-532.
Major, Louis, Theocharis Kyriacou, and O. Pearl Brereton

[S79] "Systematic literature review: teaching novices programming using robots." IET software6.6 (2012): 502-513.

Dieste, O., \& Juristo, N. (2011). Systematic review and

[S80] aggregation of empirical studies on elicitation

techniques. Software Engineering, IEEE Transactions on, 37(2), 283-304.

Prikladnicki, Rafael, Jorge Luis Nicolas Audy, and Forrest Shull.

[S81] "Patterns in effective distributed software development." Software, IEEE 27.2 (2010): 12-15.

MacDonell, S., Shepperd, M., Kitchenham, B., \& Mendes, E.

[S82] (2010). How reliable are systematic reviews in empirical software engineering?. Software Engineering, IEEE Transactions on, 36(5), 676-687.

Hart, M. D. (2008). Informatics competency and development

[S83] within the US nursing population workforce: a systematic literature review. Computers Informatics Nursing, 26(6), 320-329.

Ivarsson, M., \& Gorschek, T. (2011). A method for evaluating rigor

[S84] and industrial relevance of technology evaluations. Empirical Software Engineering, 16(3), 365-395

Morales, J. L., Sánchez, P., \& Alonso, D. (2012). A systematic

[S85] literature review of the Teleo-Reactive paradigm. Artificial Intelligence Review, 1-20.

Montagud, S., Abrahão, S., \& Insfran, E. (2012). A systematic

[S86] review of quality attributes and measures for software product lines. Software Quality Journal, 20(3-4), 425-486.

Yue, T., Briand, L. C., \& Labiche, Y. (2011). A systematic review

[S87] of transformation approaches between user requirements and analysis models.Requirements Engineering, 16(2), 75-99.

Steinmacher, I., Chaves, A. P., \& Gerosa, M. A. (2012). Awareness

[S88] support in distributed software development: A systematic review and mapping of the literature. Computer Supported Cooperative Work (CSCW), 22(2-3), 113-158.

Ardal, C., Alstadsæter, A., \& Røttingen, J. A. (2011). Common

[S89] characteristics of open source software development and applicability for drug discovery: a systematic review. Health Research Policy and Systems, 9(36), 1-16.

Dieste, O., Grimán, A., \& Juristo, N. (2009). Developing search

[S90] strategies for detecting relevant experiments. Empirical Software Engineering, 14(5), 513-539.

Šmite, D., Wohlin, C., Gorschek, T., \& Feldt, R. (2010). Empirical

[S91] evidence in global software engineering: a systematic

review. Empirical software engineering, 15(1), 91-118.

Gu, Q., \& Lago, P. (2009). Exploring service-oriented system

[S92] engineering challenges: a systematic literature review. Service Oriented Computing and Applications, 3(3), 171-188.

Zhu, M., Wang, A. I., \& Guo, H. (2012). From 101 to nnn: a review

[S93] and a classification of computer game architectures. Multimedia systems, 19(3), 183-197.

Hulaas, J., \& Binder, W. (2008). Program transformations for light-weight cpu accounting and control in the java virtual

[S94] machine. Higher-Order and Symbolic Computation, 21(1-2), 119-146.

Kitchenham, B. A., Brereton, P., Turner, M., Niazi, M. K.

[S95] Linkman, S., Pretorius, R., \& Budgen, D. (2010). Refining the systematic literature review process - two participant-observer case studies. Empirical Software Engineering, 15(6), 618-653.

Da Silva, F. Q., Suassuna, M., França, A. C. C., Grubb, A. M., Gouveia, T. B., Monteiro, C. V., \& dos Santos, I. E. (2012).

[S96] Replication of empirical studies in software engineering research: a systematic mapping study.Empirical Software Engineering, 19(3), 501-557.

Martínez-Ruiz, T., Münch, J., García, F., \& Piattini, M. (2012).

[S97] Requirements and constructors for tailoring software processes: a systematic literature review. Software Quality Journal, 20(1), 229-260.

[S98] Pino, F. J., García, F., \& Piattini, M. (2008). Software process 


\begin{tabular}{|l|l|}
\hline & $\begin{array}{l}\text { improvement in small and medium software enterprises: a } \\
\text { systematic review. Software Quality Journal, 16(2), 237-261. }\end{array}$ \\
\hline$[\mathrm{S} 99]$ & $\begin{array}{l}\text { Shuaibu, B. M., Norwawi, N. M., Selamat, M. H., \& Al-Alwani, A. } \\
(2012) . \text { Systematic review of web application security } \\
\text { development model. Artificial Intelligence Review, 1-18. }\end{array}$ \\
\hline [S100] & $\begin{array}{l}\text { Ivarsson, Martin, and Tony Gorschek. "Technology transfer } \\
\text { decision support in requirements engineering research: a } \\
\text { systematic review of REj."Requirements engineering 14.3 (2009): } \\
155-175 .\end{array}$ \\
\hline [S101] & $\begin{array}{l}\text { Catal, Cagatay, and Deepti Mishra. "Test case prioritization: a } \\
\text { systematic mapping study." Software Quality Journal 21.3 (2012): } \\
445-478 .\end{array}$ \\
\hline [S102] $]$ & $\begin{array}{l}\text { Kelly, Diane, and Cassidy R. Sugimoto. "A systematic review of } \\
\text { interactive information retrieval evaluation studies, } \\
1967-2006 . " \text { Journal of the American Society for Information } \\
\text { Science and Technology 64.4 (2012): 745-770. }\end{array}$ \\
\hline
\end{tabular}

TABLE V: LIST OF SELECTED PAPERS' KEYWORDS.

\begin{tabular}{|c|c|c|c|c|c|}
\hline [KW1] & $\begin{array}{l}\text { Systematic } \\
\text { review }\end{array}$ & $\begin{array}{l}5 \\
6\end{array}$ & [KW142] & $\begin{array}{l}\text { inference } \\
\text { strategies }\end{array}$ & 1 \\
\hline [KW2] & $\begin{array}{l}\text { Software } \\
\text { engineering }\end{array}$ & $\begin{array}{l}1 \\
1\end{array}$ & [KW143] & $\begin{array}{l}\text { inferring } \\
\text { information }\end{array}$ & 1 \\
\hline [KW3] & Ontology & 7 & [KW144] & Informatics & 1 \\
\hline [KW4] & Empirical studies & 6 & [KW145] & $\begin{array}{l}\text { information } \\
\text { security }\end{array}$ & 1 \\
\hline [KW5] & $\begin{array}{l}\text { Evidence-based } \\
\text { software } \\
\text { engineering } \\
\end{array}$ & 6 & [KW146] & $\begin{array}{l}\text { Information } \\
\text { services }\end{array}$ & 1 \\
\hline [KW6] & Mapping study & 6 & [KW147] & $\begin{array}{l}\text { information } \\
\text { systems }\end{array}$ & 1 \\
\hline [KW7] & $\begin{array}{l}\begin{array}{l}\text { Requirements } \\
\text { engineering }\end{array} \\
\end{array}$ & 6 & [KW148] & Innovation & 1 \\
\hline [KW8] & \begin{tabular}{|l} 
Empirical \\
software \\
engineering
\end{tabular} & 5 & [KW149] & $\begin{array}{l}\text { Instructional } \\
\text { strategy }\end{array}$ & 1 \\
\hline [KW9] & Quality attributes & 4 & [KW150] & Intention to leave & 1 \\
\hline [KW10] & Security & 4 & [KW151] & Internet & 1 \\
\hline [KW11] & Software process & 4 & [KW152] & $\begin{array}{l}\text { Intrusion } \\
\text { detection }\end{array}$ & 1 \\
\hline [KW12] & \begin{tabular}{l|} 
Software Process \\
Improvement
\end{tabular} & 4 & [KW153] & $\begin{array}{l}\text { ISO/IEC } 15504 \\
\text { (SPICE) }\end{array}$ & 1 \\
\hline [KW13] & $\begin{array}{l}\text { Software product } \\
\text { line }\end{array}$ & 4 & [KW154] & IT personnel & 1 \\
\hline [KW14] & agile & 2 & [KW155] & $\begin{array}{l}\text { IT Service } \\
\text { Management } \\
\text { (ITSM) }\end{array}$ & 1 \\
\hline [KW15] & Case study & 3 & [KW156] & Java & 1 \\
\hline [KW16] & $\begin{array}{l}\text { model-driven } \\
\text { engineering }\end{array}$ & 3 & [KW157] & $\begin{array}{l}\text { Large-scale } \\
\text { systems }\end{array}$ & 1 \\
\hline [KW17] & Modelling & 3 & [KW158] & $\begin{array}{l}\text { Learning software } \\
\text { organization }\end{array}$ & 1 \\
\hline [KW18] & $\begin{array}{l}\begin{array}{l}\text { Software } \\
\text { architecture }\end{array} \\
\end{array}$ & 3 & [KW159] & Life cycle & 1 \\
\hline [KW19] & $\begin{array}{l}\begin{array}{l}\text { Software } \\
\text { development }\end{array} \\
\end{array}$ & 3 & [KW160] & Machine learning & 1 \\
\hline [KW20] & Tertiary study & 3 & [KW161] & management & 1 \\
\hline [KW21] & Traceability & 3 & [KW162] & $\begin{array}{l}\text { Management } \\
\text { science }\end{array}$ & 1 \\
\hline [KW22] & UML & 3 & [KW163] & Manual search & 1 \\
\hline [KW23] & aggregation & 2 & [KW164] & MDE & 1 \\
\hline [KW24] & $\begin{array}{l}\text { Automated } \\
\text { search }\end{array}$ & 2 & [KW165] & $\begin{array}{l}\text { Measurement } \\
\text { framework }\end{array}$ & 1 \\
\hline [KW25] & Broad search & 2 & [KW166] & $\begin{array}{l}\text { metrics/measurem } \\
\text { ent }\end{array}$ & 1 \\
\hline [KW26] & Clinical research & 2 & [KW167] & Model based clone & 1 \\
\hline [KW27] & Communication & 2 & [KW168] & Model quality & 1 \\
\hline [KW28] & Cooperation & 2 & [KW169] & $\begin{array}{l}\text { Model-driven } \\
\text { development }\end{array}$ & 1 \\
\hline
\end{tabular}

\begin{tabular}{|c|c|c|c|c|c|}
\hline [KW29] & Cost estimation & 2 & [KW170] & $\begin{array}{l}\text { model-driven } \\
\text { software } \\
\text { development }\end{array}$ & 1 \\
\hline [KW30] & Domain analysis & 2 & [KW171] & Models & 1 \\
\hline [KW31] & $\begin{array}{l}\text { Empirical } \\
\text { evidence }\end{array}$ & 2 & [KW172] & Motivation & 1 \\
\hline [KW32] & $\begin{array}{l}\text { Information } \\
\text { technology }\end{array}$ & 2 & [KW173] & Motivation & 1 \\
\hline [KW33] & $\begin{array}{l}\text { Knowledge } \\
\text { management }\end{array}$ & 2 & [KW174] & $\begin{array}{l}\text { Multi product } \\
\text { lines }\end{array}$ & 1 \\
\hline [KW34] & Learningsystems & 2 & [KW175] & Natural language & 1 \\
\hline [KW35] & Mapping study & 2 & [KW176] & $\begin{array}{l}\text { Natural language } \\
\text { processing } \\
\text { systems }\end{array}$ & 1 \\
\hline [KW36] & Measurement & 2 & [KW177] & neglected diseases & 1 \\
\hline [KW37] & Measures & 2 & [KW178] & $\begin{array}{l}\text { Non-functional } \\
\text { system properties }\end{array}$ & 1 \\
\hline [KW38] & Mixed-methods & 2 & [KW179] & Nursing skills & 1 \\
\hline [KW39] & $\begin{array}{l}\text { Open source } \\
\text { software }\end{array}$ & 2 & [KW180] & $\begin{array}{l}\text { Offshore software } \\
\text { development } \\
\text { outsourcing } \\
\text { (OSDO) }\end{array}$ & 1 \\
\hline [KW40] & $\begin{array}{l}\text { People } \\
\text { management }\end{array}$ & 2 & [KW181] & $\begin{array}{l}\text { Ontology } \\
\text { Application }\end{array}$ & 1 \\
\hline [KW41] & Quality & 2 & [KW182] & $\begin{array}{l}\text { Ontology-Based } \\
\text { Knowledge } \\
\text { Management }\end{array}$ & 1 \\
\hline [KW42] & $\begin{array}{l}\text { Quality } \\
\text { evaluation } \\
\text { process }\end{array}$ & 2 & [KW183] & open source & 1 \\
\hline [KW43] & \begin{tabular}{|l|} 
Regression \\
testing
\end{tabular} & 2 & [KW184] & Organizations & 1 \\
\hline [KW44] & Routing & 2 & [KW185] & pair programming & 1 \\
\hline [KW45] & Search strategy & 2 & [KW186] & $\begin{array}{l}\text { performance } \\
\text { measures }\end{array}$ & 1 \\
\hline [KW46] & Software & 2 & [KW187] & Personality & 1 \\
\hline [KW47] & \begin{tabular}{|l|}
$\begin{array}{l}\text { Software fault } \\
\text { prediction }\end{array}$ \\
\end{tabular} & 2 & [KW188] & $\begin{array}{l}\text { pharmaceutical } \\
\text { innovation }\end{array}$ & 1 \\
\hline [KW48] & Software quality & 2 & [KW189] & physical layer & 1 \\
\hline [KW49] & $\begin{array}{l}\text { Strategic release } \\
\text { planning models }\end{array}$ & 2 & [KW190] & PL & 1 \\
\hline [KW50] & Test selection & 2 & [KW191] & Plan-driven & 1 \\
\hline [KW51] & $\begin{array}{l}\begin{array}{l}\text { Trade-off } \\
\text { approaches }\end{array} \\
\end{array}$ & 2 & [KW192] & Practical problems & 1 \\
\hline [KW52] & Variability & 2 & [KW193] & $\begin{array}{l}\text { Prevention } \\
\text { appraisal failure } \\
\text { cost scheme } \\
\end{array}$ & 1 \\
\hline [KW53] & Abstracting & 1 & [KW194] & privacy & 1 \\
\hline [KW54] & access control & 1 & [KW195] & $\begin{array}{l}\text { Problem based } \\
\text { learning }\end{array}$ & 1 \\
\hline [KW55] & $\begin{array}{l}\text { Actionable alert } \\
\text { identification }\end{array}$ & 1 & [KW196] & $\begin{array}{l}\text { Process } \\
\text { implementation } \\
\text { and change } \\
\end{array}$ & 1 \\
\hline [KW56] & $\begin{array}{l}\text { Actionable alert } \\
\text { prediction }\end{array}$ & 1 & [KW197] & Process lines & 1 \\
\hline [KW57] & $\begin{array}{l}\begin{array}{l}\text { Agile software } \\
\text { development }\end{array} \\
\end{array}$ & 1 & [KW198] & $\begin{array}{l}\text { process } \\
\text { measurement }\end{array}$ & 1 \\
\hline [KW58] & alignment & 1 & [KW199] & Process tailoring & 1 \\
\hline [KW59] & Analysis model & 1 & [KW200] & $\begin{array}{l}\begin{array}{l}\text { Product } \\
\text { development }\end{array} \\
\end{array}$ & 1 \\
\hline [KW60] & $\mathrm{AOM}$ & 1 & [KW201] & $\begin{array}{l}\text { Product line } \\
\text { engineering }\end{array}$ & 1 \\
\hline [KW61] & Application layer & 1 & [KW202] & $\begin{array}{l}\text { program } \\
\text { comprehension }\end{array}$ & 1 \\
\hline [KW62] & $\begin{array}{l}\text { Architecture } \\
\text { analysis }\end{array}$ & 1 & [KW203] & $\begin{array}{l}\text { Program } \\
\text { transformations }\end{array}$ & 1 \\
\hline
\end{tabular}




\begin{tabular}{|c|c|c|c|c|c|}
\hline [KW63] & $\begin{array}{l}\text { Architecture } \\
\text { evolution }\end{array}$ & 1 & [KW204] & \begin{tabular}{|l} 
Project \\
management
\end{tabular} & 1 \\
\hline [KW64] & $\begin{array}{l}\text { Artificial } \\
\text { intelligence }\end{array}$ & 1 & [KW205] & Proprietary & 1 \\
\hline [KW65] & aspect modelling & 1 & [KW206] & Quality costs & 1 \\
\hline [KW66] & $\begin{array}{l}\text { aspect } \\
\text { represetations }\end{array}$ & 1 & [KW207] & $\begin{array}{l}\text { Quasi-gold } \\
\text { standard }\end{array}$ & 1 \\
\hline [KW67] & aspects & 1 & [KW208] & Reactive systems & 1 \\
\hline [KW68] & aspectual UML & 1 & [KW209] & $\begin{array}{l}\text { Reconciliation } \\
\text { among } \\
\text { development } \\
\text { models }\end{array}$ & 1 \\
\hline [KW69] & $\begin{array}{l}\text { Automated static } \\
\text { analysis }\end{array}$ & 1 & [KW210] & Replications & 1 \\
\hline [KW70] & Awareness & 1 & [KW211] & Requirements & 1 \\
\hline [KW71] & BPM & 1 & [KW212] & $\begin{array}{l}\text { Requirements } \\
\text { elicitation }\end{array}$ & 1 \\
\hline [KW72] & Business models & 1 & [KW213] & $\begin{array}{l}\text { Requirements } \\
\text { selection factors }\end{array}$ & 1 \\
\hline [KW73] & $\begin{array}{l}\text { Business process } \\
\text { management }\end{array}$ & 1 & [KW214] & Research & 1 \\
\hline [KW74] & $\begin{array}{l}\text { Bytecode } \\
\text { engineering }\end{array}$ & 1 & [KW215] & research methods & 1 \\
\hline [KW75] & \begin{tabular}{|l|} 
Capability \\
Maturity Model
\end{tabular} & 1 & [KW216] & $\begin{array}{l}\text { Research } \\
\text { synthesis } \\
\end{array}$ & 1 \\
\hline [KW76] & \begin{tabular}{|l|} 
Case study, \\
computing \\
education
\end{tabular} & 1 & [KW217] & $\begin{array}{l}\text { Resource } \\
\text { management }\end{array}$ & 1 \\
\hline [KW77] & $\begin{array}{l}\begin{array}{l}\text { Change } \\
\text { characterization }\end{array} \\
\end{array}$ & 1 & [KW218] & risk management & 1 \\
\hline [KW78] & Characteristics & 1 & [KW219] & Road-mapping & 1 \\
\hline [KW79] & Cloud computing & 1 & [KW220] & Robustness & 1 \\
\hline [KW80] & Clustering & 1 & [KW221] & Scrum & 1 \\
\hline [KW81] & CMM & 1 & [KW222] & Search engines & 1 \\
\hline [KW82] & CMMI & 1 & [KW223] & $\begin{array}{l}\text { Search-based } \\
\text { software testing }\end{array}$ & 1 \\
\hline [KW83] & Code generation & 1 & [KW224] & \begin{tabular}{|l}
$\begin{array}{l}\text { Secure } \\
\text { development }\end{array}$ \\
\end{tabular} & 1 \\
\hline [KW84] & Commercial & 1 & [KW225] & \begin{tabular}{|l}
$\begin{array}{l}\text { Security } \\
\text { engineering }\end{array}$ \\
\end{tabular} & 1 \\
\hline [KW85] & $\begin{array}{l}\text { Communication } \\
\text { and } \\
\text { collaboration } \\
\end{array}$ & 1 & [KW226] & $\begin{array}{l}\text { Security } \\
\text { requirements }\end{array}$ & 1 \\
\hline [KW86] & $\begin{array}{l}\text { Competency ass } \\
\text { essment }\end{array}$ & 1 & [KW227] & \begin{tabular}{|l} 
Security \\
requirements \\
engineering \\
\end{tabular} & 1 \\
\hline [KW87] & \begin{tabular}{|l} 
Component \\
based software \\
engineering
\end{tabular} & 1 & [KW228] & Semantic clones & 1 \\
\hline [KW88] & Computer crime & 1 & [KW229] & $\begin{array}{l}\text { Service-based } \\
\text { application }\end{array}$ & 1 \\
\hline [KW89] & Computer game & 1 & [KW230] & $\begin{array}{l}\text { Service-based } \\
\text { systems }\end{array}$ & 1 \\
\hline [KW90] & $\begin{array}{l}\text { Computer } \\
\text { science }\end{array}$ & 1 & [KW231] & \begin{tabular}{|l|} 
Service-oriented \\
system \\
engineering
\end{tabular} & 1 \\
\hline [KW91] & $\begin{array}{l}\text { Computer } \\
\text { software }\end{array}$ & 1 & [KW232] & $\begin{array}{l}\text { Small and medium } \\
\text { software } \\
\text { enterprises }\end{array}$ & 1 \\
\hline [KW92] & $\begin{array}{l}\text { Computing } \\
\text { education }\end{array}$ & 1 & [KW233] & SMEs & 1 \\
\hline [KW93] & \begin{tabular}{|l|} 
Conceptual \\
Model Quality \\
\end{tabular} & 1 & [KW234] & SOA & 1 \\
\hline [KW94] & $\begin{array}{l}\text { Conceptual } \\
\text { Models }\end{array}$ & 1 & [KW235] & Software changes & 1 \\
\hline [KW95] & Control syste & 1 & [KW236] & Software clone & 1 \\
\hline [KW96] & $\begin{array}{l}\text { Control system } \\
\text { synthesis }\end{array}$ & 1 & [KW237] & $\begin{array}{l}\text { software } \\
\text { complexity }\end{array}$ & 1 \\
\hline [KW97] & $\begin{array}{l}\text { Controlled } \\
\text { experiment }\end{array}$ & 1 & [KW238] & $\begin{array}{l}\text { software cost } \\
\text { estimation }\end{array}$ & 1 \\
\hline [KW98] & Cost benefit & 1 & [KW239] & Software design & 1 \\
\hline
\end{tabular}

\begin{tabular}{|c|c|c|c|c|c|}
\hline & analysis & & & & \\
\hline [KW99] & cost prediction & 1 & [KW240] & software effort & 1 \\
\hline [KW100] & cross curting & 1 & [KW241] & $\begin{array}{l}\begin{array}{l}\text { Software effort } \\
\text { estimation }\end{array} \\
\end{array}$ & 1 \\
\hline [KW101] & CS education & 1 & [KW242] & $\begin{array}{l}\text { software } \\
\text { estimation }\end{array}$ & 1 \\
\hline [KW102] & Design & 1 & [KW243] & \begin{tabular}{|l|}
$\begin{array}{l}\text { Software } \\
\text { evolution }\end{array}$ \\
\end{tabular} & 1 \\
\hline [KW103] & $\begin{array}{l}\text { Development } \\
\text { lifecycle }\end{array}$ & 1 & [KW244] & $\begin{array}{l}\text { Software } \\
\text { evolvability }\end{array}$ & 1 \\
\hline [KW104] & diagrams & 1 & [KW245] & $\begin{array}{l}\begin{array}{l}\text { Software } \\
\text { maintenance }\end{array} \\
\end{array}$ & 1 \\
\hline [KW105] & $\begin{array}{l}\text { Distributed } \\
\text { software } \\
\text { development }\end{array}$ & 1 & [KW246] & Software metric & 1 \\
\hline [KW106] & $\begin{array}{l}\text { Distributed } \\
\text { software } \\
\text { engineering }\end{array}$ & 1 & [KW247] & $\begin{array}{l}\text { Software product } \\
\text { lines }\end{array}$ & 1 \\
\hline [KW107] & $\begin{array}{l}\text { distributed } \\
\text { software projects }\end{array}$ & 1 & [KW248] & $\begin{array}{l}\text { Software } \\
\text { robustness }\end{array}$ & 1 \\
\hline [KW108] & $\begin{array}{l}\text { Domain } \\
\text { modeling }\end{array}$ & 1 & [KW249] & Software testing & 1 \\
\hline [KW109] & Domain scoping & 1 & [KW250] & SOSE challenge & 1 \\
\hline [KW110] & drug discovery & 1 & [KW251] & SOVRM & 1 \\
\hline [KW111] & dynamic analysis & 1 & [KW252] & SPI & 1 \\
\hline [KW112] & Dynamic binding & 1 & [KW253] & $\begin{array}{l}\begin{array}{l}\text { Stakeholder } \\
\text { identification }\end{array} \\
\end{array}$ & 1 \\
\hline [KW113] & Education & 1 & [KW254] & Statecharts & 1 \\
\hline [KW114] & $\begin{array}{l}\text { Electric power } \\
\text { distribution } \\
\end{array}$ & 1 & [KW255] & Survey & 1 \\
\hline [KW115] & Electronic mail & 1 & [KW256] & \begin{tabular}{|l|} 
Symbolic \\
regression
\end{tabular} & 1 \\
\hline [KW116] & $\begin{array}{l}\text { Elicitation } \\
\text { methods }\end{array}$ & 1 & [KW257] & $\begin{array}{l}\text { Systematic } \\
\text { mapping }\end{array}$ & 1 \\
\hline [KW117] & \begin{tabular}{|l|}
$\begin{array}{l}\text { Employee } \\
\text { retention }\end{array}$ \\
\end{tabular} & & [KW258] & \begin{tabular}{|l|}
$\begin{array}{l}\text { Systematic } \\
\text { mapping study }\end{array}$ \\
\end{tabular} & 1 \\
\hline [KW118] & $\begin{array}{l}\text { Employee } \\
\text { turnover }\end{array}$ & 1 & [KW259] & $\begin{array}{l}\begin{array}{l}\text { Systematic review } \\
\text { software effort }\end{array} \\
\end{array}$ & 1 \\
\hline [KW119] & $\begin{array}{l}\text { Energy } \\
\text { management }\end{array}$ & 1 & [KW260] & Targeted search & 1 \\
\hline [KW120] & Planning & 1 & [KW261] & Taxonomies & 1 \\
\hline [KW121] & $\begin{array}{l}\text { Enterprise } \\
\text { evolution }\end{array}$ & 1 & [KW262] & $\begin{array}{l}\text { Technology } \\
\text { evaluation }\end{array}$ & 1 \\
\hline [KW122] & $\begin{array}{l}\text { Enterprise } \\
\text { Systems }\end{array}$ & 1 & [KW263] & $\begin{array}{l}\text { Technology } \\
\text { transfer }\end{array}$ & 1 \\
\hline [KW123] & $\begin{array}{l}\text { Evaluation and } \\
\text { analysis }\end{array}$ & 1 & [KW264] & $\begin{array}{l}\text { Teleo-Reactive } \\
\text { formalism }\end{array}$ & 1 \\
\hline [KW124] & $\begin{array}{l}\text { Evidence-based } \\
\text { practice }\end{array}$ & 1 & [KW265] & $\begin{array}{l}\begin{array}{l}\text { Test case } \\
\text { prioritization }\end{array} \\
\end{array}$ & 1 \\
\hline [KW125] & $\begin{array}{l}\text { Evolvability } \\
\text { analysis }\end{array}$ & 1 & [KW266] & Testing & 1 \\
\hline [KW126] & $\begin{array}{l}\text { Experimental } \\
\text { software } \\
\text { engineering }\end{array}$ & 1 & [KW267] & Tools & 1 \\
\hline [KW127] & $\begin{array}{l}\text { Experimental } \\
\text { study }\end{array}$ & 1 & [KW268] & Transformation & 1 \\
\hline [KW128] & experimentation & 1 & [KW269] & $\begin{array}{l}\text { UML state } \\
\text { machines }\end{array}$ & 1 \\
\hline [KW129] & Experiments & 1 & [KW270] & $\begin{array}{l}\begin{array}{l}\text { Unactionable alert } \\
\text { mitigation }\end{array} \\
\end{array}$ & 1 \\
\hline [KW130] & $\begin{array}{l}\text { Extreme } \\
\text { programming }\end{array}$ & 1 & [KW271] & $\begin{array}{l}\text { Unified Modeling } \\
\text { Language UML } \\
\end{array}$ & 1 \\
\hline [KW131] & fingerprinting & 1 & [KW272] & Usability & 1 \\
\hline [KW132] & $\begin{array}{l}\text { Finite state } \\
\text { machines }\end{array}$ & 1 & [KW273] & Usefulness & 1 \\
\hline [KW133] & $\begin{array}{l}\text { Free/open source } \\
\text { software }\end{array}$ & 1 & [KW274] & \begin{tabular}{|l} 
Variability \\
management
\end{tabular} & 1 \\
\hline [KW134] & $\begin{array}{l}\text { Genetic } \\
\text { programming }\end{array}$ & 1 & [KW275] & $\begin{array}{l}\text { Variant-rich } \\
\text { processes }\end{array}$ & 1 \\
\hline [KW135] & Global software & 1 & [KW276] & Warning & 1 \\
\hline
\end{tabular}




\begin{tabular}{|c|c|c|c|c|c|}
\hline & engineering & & & prioritization & \\
\hline [KW136] & group projects & 1 & [KW277] & Wave filters & 1 \\
\hline [KW137] & $\begin{array}{l}\text { GWG (game } \\
\text { world graph) }\end{array}$ & 1 & [KW278] & Web engineering & 1 \\
\hline [KW138] & Human errors & 1 & [KW279] & wireless device & 1 \\
\hline [KW139] & Identification & 1 & [KW280] & World Wide Web & 1 \\
\hline [KW140] & Industry & 1 & [KW281] & WSN & 1 \\
\hline [KW141] & $\begin{array}{l}\text { inference } \\
\text { protection }\end{array}$ & 1 & & & \\
\hline
\end{tabular}

\section{APPENDIX}

In this appendix, we present three tables. The first shows the number of selected papers per journal from 2006 to 2012; the second lists the selected papers from 2006 to 2012; and the third is a list of all keywords of the selected papers from 2006 to 2012.

\section{REFERENCES}

[1] V. M. Silva, J. I. M. S. Junior, D. N. Prata, and P. Letouze, "The importance of systematic review as a scientific research method for computer science: a quantitative systematic review," International Proceedings of Economic Development and Research, vol. 78, p. 44, 2014.

[2] A. L. Porter and I. Rafols, "Is science becoming more interdisciplinary? Measuring and mapping six research fields over time," Scientometrics, vol. 81, no. 3, pp. 719-745, 2009.

[3] T. Greenhalgh, How to Read A Paper: The Basics of Evidence-Based Medicine, Wiley-Blackwell, 2006.

[4] M. Petticrew and H. Roberts, Systematic Reviews in Social Science: a Practical Guide, Blackwell Publishing, 2006.

[5] D. Tranfield, D. Denyer, and P. Smart, "Towards a methodology for developing evidence-informed management knowledge by means of systematic review," British Journal of Management, vol. 14, pp. 207-222, 2003,

[6] URM - Uniform requirements for manuscripts. (2008). International Committee of Medical Journals. [Online] Available: http://www.icmj.org

[7] H. P. Breivold, I. Crnkovik, and M. Larsson, "A systematic review of software architecture evolution research," Information and Software Technology, vol. 54, pp. 20-46, 2012.

[8] A. Ampatzoglou and I. Stamelos, "Software engineering research for computer games: a systematic review," Information and Software Technology, vol. 52, pp. 888-901, 2010.

[9] EBSE Technical Report, Guidelines for performing Systematic Literature Reviews in Software Engineering, Software Engineering Group School of Computer Science and Mathematics Keele University - UK, and Department of Computer Science University of Durham UK, Version 2.3, 2009

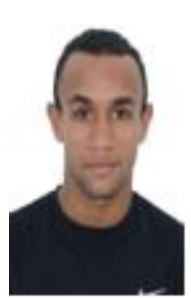

José Itamar Mendes de Souza Júnior is a graduate student of computer science at Federal University of Tocantins (UFT - "Universidade Federal do Tocantins"), medalist in the Brazilian Mathematical Olympiad public Schools (OBMEP - "Olimpíada Brasileira de Matemática das Escolas Públicas") and member of the software development nucleus (NDS - "Núcleo de Desenvolvimento de Software").



Valéria Martins Silva a graduate student of computer science at Federal University of Tocantins (UFT "Universidade Federal do Tocantins") and member of the software development nucleus (NDS - "Núcleo de Desenvolvimento de Software").

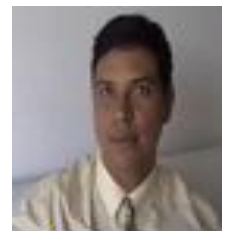

David Prata was born in Goiânia, Brazil on September 18, 1965. Dr. Prata completed his bachelor of computer science in 1992. Then on, he went to complete his specializing in academician. He worked as a system analyst to Tocantins Government, being in charge for the accountability and financial systems. Later, he successfully completed his master degree in computer science from Campina Grande Federal University, with application research in education in 2000 year. He coordinated graduate and undergraduate courses in computer science at Alagoas faculty in Maceio, Brazil. He was allotted to Federal University of Alagoas in 2006. Then, he moved to Federal University of Tocantins. His doctoral was developed in part at Carnegie Mellon University, USA completed in 2008. He is currently coordinating a master degree in computational model. His research interests are education and ecosystems.

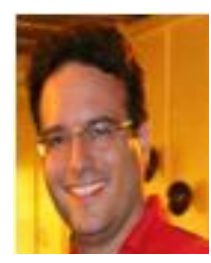

Patrick Letouze is a control and automation engineer with a master science degree on electrical engineering telecommunications, and a doctor in science degree on electrical engineering - health informatics. Currently, he is with the Computer Science Department at the Federal University of Tocantins (UFT - "Universidade Federal do Tocantins"). He is the director of the Software Development Nucleus (NDS - "Núcleo de Desenvolvimento de Software"), the elected president the of the committee of ethics in research with human beings, term 2014-2016, is vice-director of the postgraduate program in systems' computational modeling and is the former director of research of the Federal University of Tocantins. 